\title{
Novel nonlinear wave equation: regulated rogue waves and accelerated soliton solutions
}

\author{
Abhik Mukherjee \\ Department of Mathematics and Applied Mathematics, \\ University of Cape Town, Cape Town, SOUTH AFRICA \\ Anjan Kundu \\ Saha Institute of Nuclear Physics \\ Kolkata, INDIA
}

\begin{abstract}
A new exactly solvable $(1+1)$-dimensional complex nonlinear wave equation exhibiting rich analytic properties has been introduced. A rogue wave (RW), localized in space-time like Peregrine RW solution, though richer due to the presence of free parameters is discovered. This freedom allows to regulate amplitude and width of the RW as needed. The proposed equation allows also an intriguing topology changing accelerated dark soliton solution in spite of constant coefficients in the equation.
\end{abstract}

\section{INTRODUCTION}

Upsurge of interest in exactly solvable nonlinear equation in (1+1)- dimension is due to the exclusive properties of their solutions with applicable potentials. Most important of such exact solutions is the localized soliton solutions, which move with a constant velocity without any change of their shape and size. Such equations can be real ones like Korteweg de Vries (KdV) equation, modified KdV, Sine Gordon equations etc or complex like Nonlinear Schrodinger equation (NLS), DNLS, Hirota equations etc.

However in recent years, the emphasis has been shifted towards newer solutions in diverse physical applicability, with attempts to discover new nonlinear exactly solvable equations. Therefore, the focus has been concentrated gradually on more intricate solutions like accelerating solitons [1], topologically nontrivial solutions [2], RW solutions etc [4].

Though RWs are deep ocean surface waves with manifestly $(2+1)$ dimensional properties [3], the concept of RW has been penetrated recently to $(1+1)$ dimensions with a large number of experimental and theoretical investigations in a wide range of fields [4]. The most popular of such RW solutions is Peregrine breather solution. Such RWs unlike usual soliton solutions grow in amplitudes while moving reaching to its maximum at certain time and disappearing again to the background waves. However in spite of its generic nature and success in explaining RW phenomena in experimental observations in diverse fields [8 14], the description of RW by Peregrine breather is limited to a fixed maximum amplitude (3 times of background wave) and fixed RW width. This is due to the absence of any free parameters in Peregrine RW solutions [4].

Most of the subsequent findings in the systems like Kundu Ekkaus equation [5], Davey Stewartson equation [6], Hirota equation [7] etc are also devoid of free parameters in single peak RW solution. Only in higher RW solutions with multiple peaks which are physically less important such free parameters can arise [16 18]. Therefore a natural question is to search for possible one peaked RW solution for different nonlinear equation which would allow free parameters to regulate the amplitude and width to fit different types of such waves obtained in nature and real experiments.

The accelerating solitons, which is the focus of another current interest arise on the other hand in inhomogeneous media, modelled by extending the known integrable equations to their inhomogeneous forms by introducing variable dependent coefficients. However, the logical curiosity is whether accelerating solitons could appear even in a homogeneous medium described by equation with constant coefficient. This would not only enhance our understanding of the basic cause behind a variable solitonic velocity and its shape but also could open up new applicability. A continued interest in nonlinear physics is a fascinating world of topological soliton solutions. Though in one dimension, its impact is not that prominent with the appearance of stable kink solutions in Sine Gordon and $\phi^{4}$ theory and dark solitons in NLS equation, undoubtedly have drawn much attention. As we know, for obtaining kink and dark solitons and their multisoliton variants one has to use different functional form of the solutions with increasing complexity with the increase of soliton number. Therefore it would be an intriguing question to investigate whether one can generate the nature of multidark solitons using the simplest form of dark solitons, given typically by tanh functions.

Our present aim concerns the above posed natural and inquisitive questions, for investigating which we propose a novel exactly solvable nonlinear complex wave equation with current like nonlinearity. Within the framework of 
our nonlinear equation we investigate the possibility of finding controllable one peak RW solution, accelerating and topology changing dark solitons.

Proposed complex nonlinear wave equation: We introduce a novel complex wave equation

$$
\frac{\partial^{2}}{\partial T^{2}} \psi-\frac{\partial^{2}}{\partial X^{2}} \psi=2 i \psi\left(J_{X}+J_{T}\right)
$$

along with its complex conjugate, Where $J_{X}=\psi^{*} \frac{\partial \psi}{\partial X}-\psi \frac{\partial \psi^{*}}{\partial X}$ and $J_{T}=\psi^{*} \frac{\partial \psi}{\partial T}-\psi \frac{\partial \psi^{*}}{\partial T}$ are current like nonlinearities. This equation in the lightcone coordinates $(x=(T+X) / 2, t=(T-X) / 2)$ takes the simpler form

$$
\psi_{x t}+2 i \psi\left(\psi \psi_{x}^{*}-\psi^{*} \psi_{x}\right)=0
$$

where the subscripts denote partial derivatives. We show that, the equation (2) allows a richer set of exact solutions related to some important questions posed above. It is noticeable that (1) is a dispersionless equation and allows nontrivial boundary condition at space infinities $(|\psi| \rightarrow$ const., $\quad|x| \rightarrow \infty)$, which will be significant for our investigation. We use both forms (1) and (2) of our equation whichever is convenient. We focus below on richer RW and dark soliton solutions of the proposed exactly solvable nonlinear PDE.

\section{CONTROLLABLE ROGUE WAVE SOLUTIONS:}

Such sudden and steep isolated waves with a single peak, borrowing the concept from the deep oceanic waves, are causing upsurge of theoretical and experimental studies in various fields [4]-[14]. Such phenomena can be modelled by RW solutions defined in $(1+1)$ space-time dimensions.

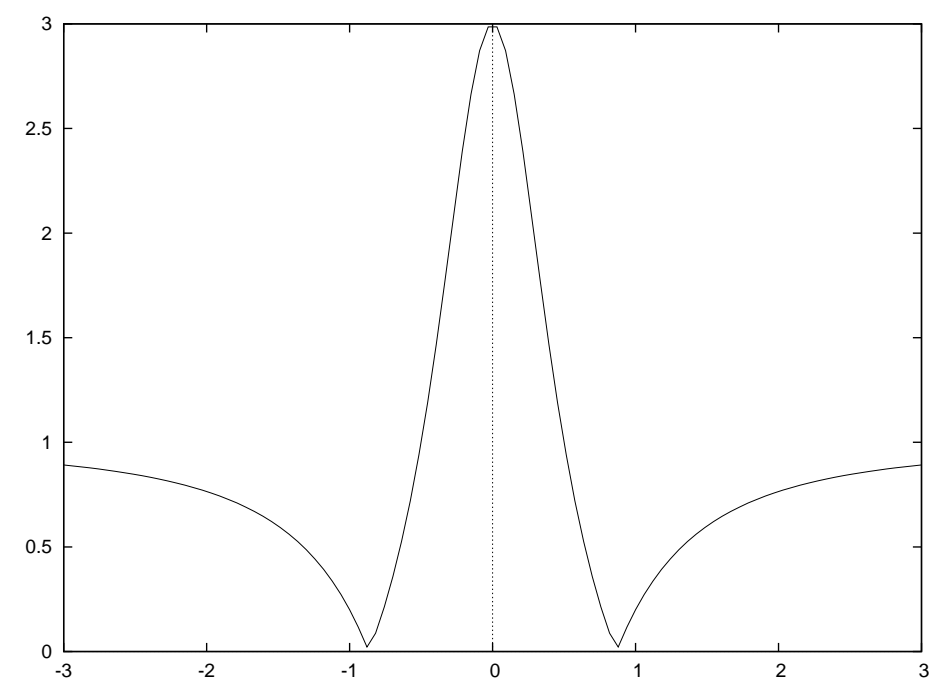

FIG. 1: Amplitude variation of the full grown 1D rogue wave, modelled by the modulus of the static Peregrine breather . The maximum amplitude 3 is attained at $x=0$, while it goes to its asymptotic value 1 at $x \rightarrow \pm \infty$. The maximum inclination attainable is $3 \sqrt{3}$ at $x=\frac{\sqrt{3}}{6}$, and becomes 0 both at $x=0$ and $x \rightarrow \pm \infty$.

Therefore, Peregrine breather which offers such a simple solution (see Fig .1) became the most popular RW model. However, as we see from the figure the solution allows only fixed maximum amplitude, shape and width for the RW. Since, in diverse situations observed experimentally the single peak RW can appear in diverse amplitude, width and shape the application of Peregrine solution becomes a bit restricted with a natural need for having richer RW solutions allowing adjustable form of such solutions. Our aim here is to offer such a general solution obtained from our proposed equation.

Analyzing our nonlinear wave equation (1) and with the physical input that at space and time infinities the RW solutions would go to linear background waves $e^{-2 i(X-T)}$, while its amplitude will grow nonlinearly and reach its maximum at the centre, we can extract the exact RW solution in the explicit form

$$
\psi=e^{-2 i(X-T)}\left[-1+\frac{1+2 i(X-T)}{c+(T-X)^{2}+\frac{\alpha}{4}(X+T)^{2}}\right],
$$




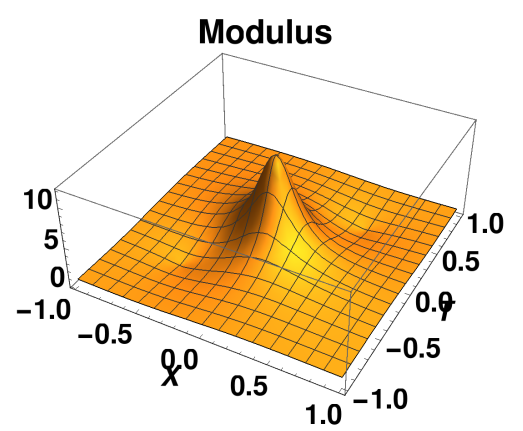

(a)

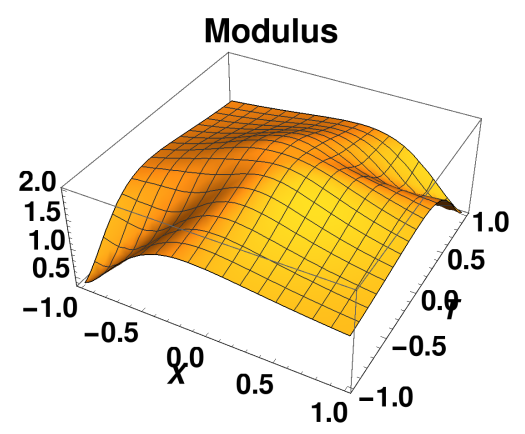

(c)

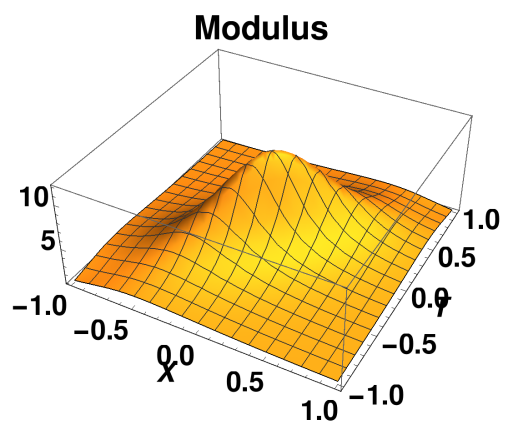

(b)

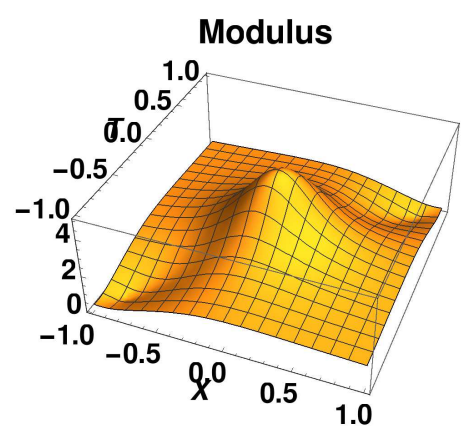

(d)

FIG.1: Rogue wave (RW) modelled by the modulus of our solution (3) with different shapes and sizes, generated from the same single peak solution. (a)High amplitude and high steepness are obtained for $\alpha=4, c=\frac{1}{13}$. (b)High amplitude and low steepness are obtained for $\alpha=0.4, c=\frac{1}{13}$. (c)Low amplitude and low steepness are obtained for $\alpha=0.4, c=\frac{1}{3}$. (d)Moderate amplitude and moderate steepness are obtained for $\alpha=1.2, c=\frac{1}{6}$.

It is important to note that, the above solution includes two arbitrary parameters $\alpha$ and $c$, which will be crucial for controlling the physical properties of this RW solution by changing these parameters as desired. Looking closely to solution (3), we see that at space-time infinities $|X| \rightarrow \infty, \quad|T| \rightarrow \infty$ the solution goes to the background traveling wave and at $T=X=0$, the amplitude attains its maximum value $\left(\frac{1}{c}-1\right)$. It is evident therefore that the maximum amplitude of the RW solution can be increased or decreased by varying the constant $c$ as demonstrated in Fig 2 . This is a significant positive difference from the Peregrine RW which shows the maximum amplitude fixed at 3. Similarly it reveals also that the width, inclination and shape of our RW solution (3) becomes functions of two arbitrary parameters $\alpha$ and $c$ allowing us to control the property as needed to fit the experimental observation. We mention again that such a tuning cannot be done through the well known one-peaked Peregrine RW solution ( Fig.1), due to the absence of free parameters.

Condition favorable for rogue wave solutions: modulation instability:

After finding explicit RW solution in the proposed nonlinear equation we would like to investigate the condition crucial for the sudden generation of rogue wave solution in our system out of the linear background waves, which is instigated mostly by the modulation instability suffered by the wave due to the presence of nonlinearity and dispersion in the media. This is a specific condition between the frequency and wave vector, when a small perturbation of the linear wave becomes unstable with a sudden growth in time. Such a modulation instability is supposed to be crucial in the formation of rogue wave in the physical system.

For easier calculations, we concentrate on the equivalent form (2) for deriving the instability condition, known as modulation instability for the background wave, taking it as a perturbed linear wave form: $\psi=\left(A_{0}+\epsilon(x, t)\right) e^{i(\omega t+k x)}$. Inserting it in the nonlinear equation (2) and neglecting higher powers of $\epsilon$, we can derive a linear homogeneous equation for $\epsilon$. Using the standard analysis, we can derive from this linear equation, the condition for the modulation instability suffered by such a perturbation given by the imaginary part of the modulation frequency which in our case is $\omega_{m}= \pm\left(i \sqrt{\omega^{2}-4 A_{0}^{2} \omega}\right)$.

Note that, this physical property of instability would retain the same for our original nonlinear equation (1) though 
its explicit form will change due to change of the coordinate system.

\section{ACCELERATED DARK SOLITON SOLUTIONS:}

Unlike the RW solution the soliton solutions including the kink and the dark solitons which propagates with constant velocity and amplitude without change in their shapes are more prevalent in exactly solvable nonlinear system. Therefore, our intension here is to look for the soliton solutions in the simplest form $\psi(x, t)=\rho(x, t) e^{i \theta(x, t)}$ and explore the possibility of finding the accelerated soliton in our proposed equation which would be rather an intriguing problem due to the homogeneity of the equation with constant coefficients. Inserting $\psi$ in (2) and equating real and imaginary parts, we get two coupled nonlinear equations:

$$
\begin{gathered}
\rho_{x t}-\rho \theta_{x} \theta_{t}+4 \rho^{3} \theta_{x}=0, \\
\rho_{x} \theta_{t}+\rho_{t} \theta_{x}+\rho \theta_{x t}=0,
\end{gathered}
$$

For usual soliton solutions, carrier waves are given through the phase $\theta=k x+\omega t+\theta_{0}$ with constant frequency $\omega$ and wave vector $k$. However, we explore the possibility of generalizing it nonlinearly as

$$
\theta(x, t)=k x+q(x)+(\omega t+p(t))+\theta_{0}
$$

. where $q(x), p(t)$ are arbitrary functions. Using the form (6), the second equation (5) gives

$$
\left(\omega+p^{\prime}(t)\right) \rho_{x}=-\left(k+q^{\prime}(x)\right) \rho_{t}
$$

which clearly allows the functional dependence of $\rho(\xi)$, with $\xi=\xi(x, t)$, giving

$$
\left(\omega+p^{\prime}(t)\right) \rho_{\xi} \frac{\partial \xi}{\partial x}=-\left(k+q^{\prime}(x)\right) \rho_{\xi} \frac{\partial \xi}{\partial t}
$$

Consistency gives the solution, $\xi=\nu\left(k x+q(x)-(\omega t+p(t))+\xi_{0}\right)$ keeping the same $\mathrm{x}$ dependence as in $\theta$ but negating its t dependence, with $\nu, \xi_{0}$ constants, which may be chosen as desired. It is crucial to note here, that the appearance of the arbitrary functions in the argument $\xi$ in the present case, which will be responsible for creating accelerating solitons, is an unusual property for a solitonic equations. Notice that, equation (7) coming from the imaginary part of our equation contains only derivatives of function $\rho$ which can be traced back to the appearance of arbitrary functions in $\xi$. On the other hand, the corresponding equation in the wellknown NLS case contains function $\rho$, together with its derivatives, which spoils this property and hence does not allow accelerating soliton in the standard NLS equation.

The real part of the equation (4) is simplified now using the explicit forms of $\theta$ and $\xi$ as

$$
\nu^{2} \rho_{\xi \xi}+\rho-\frac{4}{\omega+p^{\prime}(t)} \rho^{3}=0
$$

Note that, the exact solution of the above equation does not allow any function $p(t)$ other than constant. Therefore, considering the same we look for explicit solutions of (9).

On the other hand, for $\nu^{2}>0$, i.e, with real $\nu$ linked with the soliton width, equation (9) with $p(t)=$ const. turns into the canonical form admitting tanh solution representing dark soliton

$$
\rho=\frac{\sqrt{\omega}}{2} \tanh [\xi], \xi=\nu\left(k x+q(x)-\omega t+\xi_{0}\right) ., \nu= \pm \frac{1}{\sqrt{2}}
$$

The appearance of the arbitrary function $q(x)$ is a unique feature of our equation in spite of its constant coefficients which differs drastically from the usual NLS and derivative NLS families of equations. As mentioned above, the systems with inhomogeneities modelled by x-dependent functions appearing explicitly in the defining equations might show such a property of accelerated solitons found here. We show interestingly, that this freedom of choice of function $p(x)$ gives us in one hand an accelerating dark soliton and on the other hand the solution with changing topological properties. 


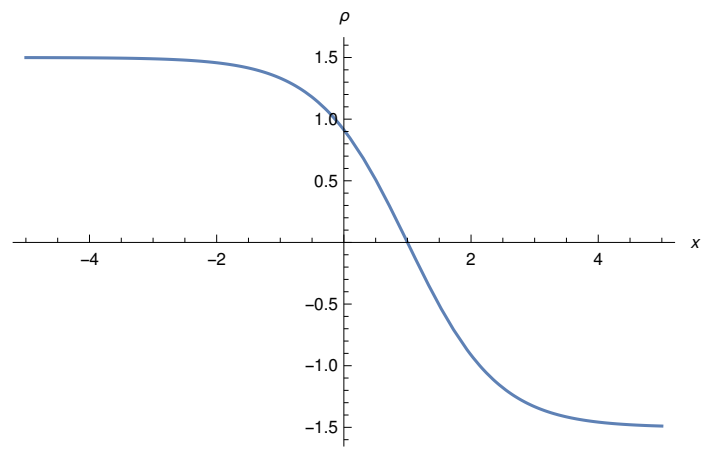

(a) $q(x)=x$

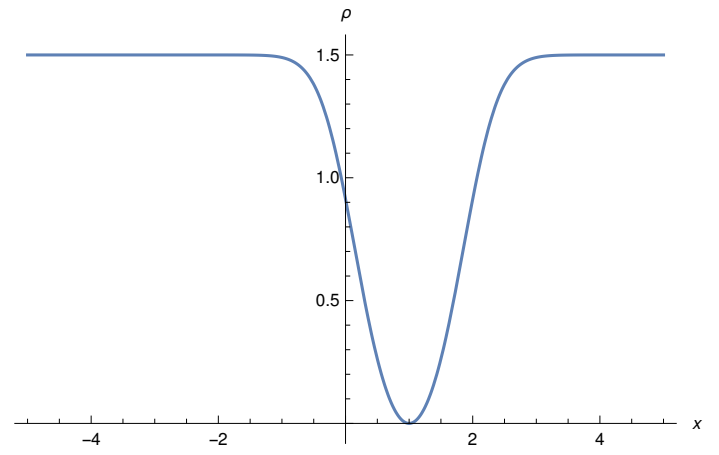

(b) $q(x)=x^{2}$

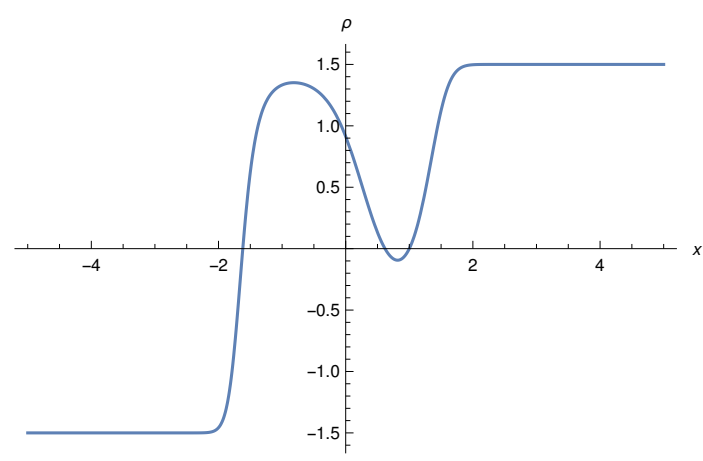

(c) $q(x)=x^{3}$

FIG.2: Accelerated soliton modelled by our solution (10) at $\mathrm{t}=0$, with different choices of arbitrary functions $q(x)$ and for the choice of constants $\mathrm{k}=-2, \mathrm{w}=3, \theta_{0}=1 ; \xi_{0}=1$.

For identifying the acceleration of soliton (10), we may track the centre of the solution at $\xi=0$. Through simple steps, we may derive the variable velocity $v(x)$ as well as the acceleration $a(x)$ as

$$
v(x)=\frac{\omega}{k+q^{\prime}(x)}, \quad a(x)=-q^{\prime \prime}(x) \frac{\omega^{2}}{\left(k+q^{\prime}(x)\right)^{3}}
$$

Obviously for $q(x)=x$, we get constant velocity solution, however for any other choice of function $q(x)$ as the structure (11) of the velocity $v(x)$ and acceleration $a(x)$ suggests that the soliton moves with a retarded velocity with the increase or decrease of $x$.

At the same time, the appearance of the arbitrary function $\mathrm{q}(\mathrm{x})$, the dark soliton solution has an intriguing effect in the topological characteristic of such soliton solutions defined through the boundary condition as $Q=(\rho(x \rightarrow$ $\infty)-\rho(x \rightarrow-\infty))$. For $q(x)=$ const. we get a typical kink or antikink solution $\rho= \pm \frac{\sqrt{\omega}}{2} \tanh [\nu(k x-\omega t]$ linked to $Q= \pm 1$, extrapolating between different vaccume. It is remarkable that, from other nonlinear choice for $q(x)$, we start getting multi kink, antikink solutions allowing change of topological properties generated from the same tanh solutions (10) which is atypical in soliton theory as well as for topological properties. The following simple examples will illustrate the procedure, shown also in Fig.3.

Case $q(x)=x^{2}$ : It is easy to see that, in this case the topological characteristic becomes trivial since $Q=\frac{1}{2}(\rho(x \rightarrow$ $\infty)-\left(\rho(x \rightarrow-\infty)=\frac{1}{2}(1-1)=0\right.$ which is due to the simultaneous appearance of kink and antikink extrapolating finally from the same vacuum. Therefore, we have created 2- solitons (kink and antikink) from the same solution (10). Recall that for standard NLS case, kink-antikink solutions get more complicated expression than tanh.

Case $q(x)=x^{3}$ : This would lead again to $Q=\frac{1}{2}\left(\rho(x \rightarrow \infty)-\left(\rho(x \rightarrow-\infty)= \pm \frac{1}{2}(1-(-1))= \pm 1\right.\right.$, showing the generation of 3- solitons (kink, antikink and kink) from the same solution (10). 


\section{CONCLUSIVE REMARKS:}

A new exactly solvable (1+1)-dimensional complex nonlinear wave equation has been introduced in this work. A rogue wave (RW) solution, localized in space-time and richer due to the presence of free parameters is discovered. This freedom allows to regulate amplitude and width of the RW as needed. The proposed equation allows also an intriguing topology changing accelerated dark soliton solution in spite of constant coefficients in the equation. This externally controllable solutions may have its useful application in various branches of science and may pave new direction of research.

[1] A.V.Gorbach and D.V Skryabin, Phys.Rev.A 76,053803,2007

[2] M.O.Katanaev, Theoretical and Mathematical Physics 132(2), 163,2004

[3] A Kundu, A Mukherjee and T naskar, Proc. R. Soc. A 470, 20130576,2014

[4] C Kharif, E Pelinovsky and A Slunyaev Rogue Waves in Ocean, (Berlin,Germany:Springer, 2009).

[5] X.Wang, B.Yang, Y.Chen and Y. Yang, Phys.Scr.89, 095210,2014

[6] Y. Ohta and J.Yang, Phys.Rev.E 86, 036604,2014

[7] A.Ankiewicz, J.M.Soto-Crespo, N.Akhmediev, Phys.Rev.E 81, 046602,2010

[8] C Bonatto, M Feyereisen, S Barland, M Giudici, C Masoller, L Rios JR and Tredicce JR, Phys.Rev.Lett.107, 053901,2011

[9] DR Solli, C Ropers, P Koonath and B Jalali, Nature 450,06402,2007

[10] M Onorato et al, Phys. Rev. Lett. 102,114502,2009

[11] A Montina, U Bortolozzo, S Residori and FT Arechhi, Phys. Rev. Lett 103,173901, 2009

[12] B Kibler et al Nat.Phys,6,790-795,2010

[13] M Shats, H Punzmann and H Xia Phys. Rev.Lett 104,104503,2010

[14] A Chabchoub, NP Hoffmann, N Akhmediev, Phys.Rev.Lett,106,204502,2011

[15] R. S Johnson, A Modern Introduction to the Mathematical Theory of Water Waves, (Cambridge University Press, Cambridge, 1997) and references therein.

[16] N. Akhmediev, A. Ankiewicz and and J.M Soto Crespo, Phys. Rev E 80, 026601 (2009).

[17] P. Dubard and and V.B Matveev, Nat Hazards Earth Syst.Sci.11 , 667 (2011).

[18] A. Ankiewicz, D.J Kedziora and N. Akhmediev, , Phys. Lett A 375, 2782 (2011).

\section{ACKNOWLEDGEMENT}

Abhik Mukherjee acknowledges his colleagues for valuable discussions. 